\title{
Provision of long term oxygen therapy
}

Domiciliary oxygen therapy has for many years been available in the United Kingdom through the family practitioner services. Hitherto the approved method of providing this has been by means of 1360 litre cylinders with regulating valves capable of delivering flows of 2 or 41 of oxygen per minute. The marked geographical variation in the utilisation of these cylinders, which is unrelated to area death rates for respiratory diseases and hence to the presumed prevalence of those conditions for which supplementary oxygen might be most needed, led Williams to question the rationale for home oxygen therapy: In most cases it is provided for occasional or short term use ${ }^{2}$ when the patient perceives a need for it, usually at times of acute breathlessness. Only in a minority has the aim been to offset the harmful consequences of chronic hypoxia, including pulmonary hypertension and polycythaemia, and so to prolong life. For this purpose, according to the best available evidence, controlled oxygen supplements are required by the patient for a total of between 12 and 24 hours daily. ${ }^{3-8}$ It is with such long term oxygen therapy (LTOT) that we are concerned here.

Providing oxygen cylinders in patients' homes on the scale needed for LTOT is both inconvenient and expensive, and there can be little doubt that in the past this has often inhibited physicians from recommending such treatment. At present the most cost effective way to provide LTOT for a patient whose home has a suitable electrical supply is with an oxygen concentrator. This was shown in an economic appraisal of domiciliary LTOT $^{9}$ and has been confirmed in a report prepared for the Department of Health and Social Security (DHSS) by the North Western Regional Health Authority (NWRHA) in Manchester after a trial introduction of concentrators in that region. ${ }^{10}$

It is now 17 years since Cooper's description of a molecular sieve oxygen concentrator suitable for clinical use. ${ }^{11}$ Within one year Cotes and his colleagues had tested a prototype in hospital ${ }^{12}$ and by 1973 Stark and Bishop had shown that a concentrator could be used successfully in the patient's home..$^{13}$ With those early, cumbersome machines there were considerable problems with reliability

Address for reprint requests: Dr TB Stretton, Manchester Royal Infirmary, Manchester M13 9WL. and they were very noisy in use. This has all changed: currently available concentrators, several of which are evaluated in two papers in this issue,,$^{14}$ is are more compact and more easily transported; they fit better into the home environment, create a noise level which is acceptable if not ideal, require relatively little maintenance and are capable of generating an adequate flow of greater than $90 \%$ oxygen for long periods of time. At the beginning of December this year the DHSS will make oxygen concentrators generally available on prescription for use in the home. Whereas the trial LTOT service which was conducted by the NWRHA was hospital based, the equipment being installed only on the recommendation of a respiratory physician, in the scheme which is about to be introduced in England and Wales concentrators will be supplied through the family practitioner services. It is sincerely to be hoped that they will be prescribed on a rational basis after careful selection of patients for whom LTOT is appropriate.

\section{Arrangements for prescription}

It is understood that the system for arranging the delivery and installation of a concentrator will work as follows. The general practitioner will make a prescription for the provision of an oxygen concentrator together with any other necessary equipment, and will indicate the duration of treatment in hours per day on form FP 10 and hand this to the patient. At the same time an explanation will be given of the arrangements which will then be put in hand. The patient will retain form FP 10 until visited by a representative of the concentrator supplier and then hand the form to the representative. Meanwhile the family doctor will notify the regional supplier by telephone of the name and whereabouts of the patient for whom the prescription has been written. The supplier will confirm in writing with the practitioner, the patient and the Family Practitioners' Committee (FPC) that the order has been received and will then proceed with the installation.

Suppliers of concentrators are to be selected by open competitive tender and one supplier only will be contracted to provide this service in any given region. For this purpose England and Wales have been divided into nine regional groups of FPCs (which do not share the same boundaries as Regional Health Authorities). FPCs will keep a record 
for each patient for whom a concentrator is provided (including a note of the prescribed regimen of usage) and will receive invoices from the supplier for the costs connected with the installation, rental, maintenance, running and ultimate removal of the concentrator. At the time of writing, the arrangements for the supply of concentrators in Scotland are unclear.

Clinical guidelines for the prescription of LTOT are being prepared by the DHSS for inclusion in the Drug Tariff. The guidelines, in their draft form, have already been reported by Williams and Nicholl $^{16}$ and they are reproduced here verbatim:

\section{Draft DHSS Guidelines for Prescription of Long term Oxygen Therapy}

Absolute indication Such patients will have chronic obstructive airways disease, hypoxaemia, and oedema (or previously witnessed oedema). Suitable patients would have a forced expiratory volume of less than $1.5 \mathrm{l}$, a forced vital capacity of less than 21 , an arterial oxygen tension less than $7.3 \mathrm{kPa}(55 \mathrm{~mm} \mathrm{Hg})$, and some elevation of arterial carbon dioxide tension greater than $6.0 \mathrm{kPa}$ or $45 \mathrm{~mm} \mathrm{Hg}$.

These tests should be made in a stable phase of the condition when all reversible factors have been fully treated, eg infection, reversible airways disease, cardiac disease. In order to document stability, the spirometric measurements and the arterial blood gases should be repeated at not less than three weeks later. There should be a variation of no more than $\pm 20 \%$ in the spirometric measurements and a variation of no more than $\pm 5 \mathrm{~mm} \mathrm{Hg}$ or $0.6 \mathrm{kPa}$ in the arterial tension. If the tests have improved by more than these limits then a further three weeks interval should elapse and the tests should be repeated.

Other patients with chronic obstructive airways disease The second group will contain patients with chronic obstructive airways disease having the same spirometric characteristics and the same levels of arterial oxygen tension as those described above, but no hypercapnia or oedema.

Many such patients were studied in American trials of long term domiciliary oxygen therapy and benefit was clearly shown but such patients were excluded from the British studies. However, many of these patients, including refractory asthmatics and sufferers from cystic fibrosis, might be expected to benefit from long term oxygen therapy. On present evidence, long term oxygen therapy should not be denied but the same criteria of stability to ensure that they are truly chronically hypoxaemic should be applied.

Palliative use of oxygen This group includes patients with other respiratory conditions associated with severe arterial hypoxaemia but without hypercapnia, for which oxygen therapy may have a useful palliative effect, without necessarily affecting survival.

Examples would be severe hypoxaemia associated with the terminal phases of fibrosing alveolitis, industrial lung fibroses, and collagen disorders and lung infiltrations with sarcoid or malignancy.
Other conditions Palliation of chronic respiratory failure associated with kyphoscoliosis, obesity, hypoventilation and the end stages of irreversible peripheral neuropathies and muscle disorders is less certain. However, some of these conditions are associated with such severe disturbances of ventilatory drive that the abolition of hypoxaemia by increased inspired oxygen may be dangerous. If oxygen therapy is considered in such patients the initial assessment would have to be more detailed than that already described for patients with chronic obstructive airways disease.

\section{Assessment of suitability for long term oxygen therapy}

If these guidelines are followed closely a formidable number of patients will warrant an evaluation of their suitability for treatment. This process requires the expertise of respiratory physicians and is time consuming. Will the available resources be able to cope with the demand? An attempt by Williams and Nicholl ${ }^{16}$ to estimate how many subjects in the community, aged over 45 years, with hypoxaemic chronic obstructive bronchitis might qualify for the prescription of a concentrator suggests that in Sheffield, where the study was based, this could be around 600, thus providing a figure for England and Wales of 60000 . Even if one takes only the lower confidence limits from that study as a guide, the number of subjects who would be "eligible" for a concentrator are about 130 for Sheffield and 13000 for England and Wales as a whole. In selecting patients with chronic airflow obstruction for LTOT the Nocturnal Oxygen Therapy Trial (NOTT) Group observed that $45 \%$ of those initially identified as candidates improved during the 4 week period of assessment and were then no longer so hypoxaemic as to warrant treatment. ${ }^{17}$ Limited personal experience of trying to select patients for LTOT is also in accordance with an "acceptance ratio" in the region of $50 \%$ (although the reasons for rejection may differ). It remains unknown how many patients general practitioners and other colleagues will refer to respiratory physicians for consideration of LTOT and what the "acceptance ratio" will be for the numbers of patients finally recommended for treatment as a percentage of those referred. Only guesswork is possible at this stage but it seems quite likely that three or four patients will need to be assessed for every concentrator finally installed in a home. If Williams and Nicholl are correct in their estimation, ${ }^{16}$ and if to the sufferers from chronic obstructive lung disease who may be "eligible" for a concentrator are added those with other diseases, the implications are obvious: the assessment of the large numbers of potential candidates for LTOT will constitute a large additional work- 
load. It may be the view of the DHSS that most of those in need of a concentrator are already receiving LTOT from oxygen cylinders and that it will be a simple matter to transfer these patients to concentrators, with a consequent financial saving, and that the introduction of LTOT will have little impact on the hospital services. On the contrary, it seems likely that even if there is not an immediate flood of requests to assess patients for their suitability for LTOT there will be a rapid growth in these. So far, however, no recommendations appear to have been made to Regional Health Authorities to make provision for this labour intensive assessment process, which is an essential component of the proposed LTOT service.

\section{Indications for long term oxygen therapy}

Deciding whether and when to recommend that a patient should embark on LTOT is not simply a matter of establishing that the patient fulfils certain pathophysiological criteria, crucially important though these are. Consideration should be given to the quality of the patient's life, and this involves making value judgements which are both difficult and imprecise. LTOT confers its own burden on the patient and the family, and requires willingness and a lasting commitment if it is to be maintained. The patient's age, the presence of other significant pathology, the social history, the attitude and capability of the spouse, or other closely involved relative, may all influence a decision to treat. Close collaboration between the family practitioner and the respiratory physician is clearly most desirable in reaching the decision.

Difficulty in deciding when to initiate LTOT is created by the uncertainty as to where patients are placed on their own, unique "natural history curve." Some do appear to be remarkably tolerant of chronic hypoxia. To introduce LTOT too early would be unacceptable but if it is to be instituted it must not be delayed until it is too late to be of benefit. The choice of oedema formation as a marker of cor pulmonale and an indicator of the need for LTOT has the great merit of being recognisable clinically and being a clearly defined stage in the evolution of the disease. Unfortunately it is a late feature and has long been linked with a poor prognosis. In the Medical Research Council (MRC) trial of LTOT $^{6}$ the cumulative five year mortality was $67 \%$ in those allocated to the control group, who did not receive LTOT but whose treatment we may assume was otherwise the best available. There was also an indication from the same trial that the most severely afflicted male patients were doomed soon to die whether they received LTOT or not; it was suggested that their (inevitable) deaths could have accounted for the paradox that improved survival did not become evident in the treated group of male patients until 500 days had elapsed. It would obviously be helpful if those with the worst outlook could be identified before LTOT is recommended. Attempts were made by the MRC Working Party to establish criteria which would permit such a prediction but the best of these, the sum of the patient's arterial carbon dioxide tension $(\mathrm{mm} \mathbf{H g}$ ) and the red cell mass $(\mathrm{ml} / \mathrm{kg})$, proved to be a poor discriminator. The finding by Ashutosh and colleagues ${ }^{18}$ that the prognosis could be predicted according to the magnitude of response in the patient's pulmonary arterial pressure after only 24 hours' oxygen therapy requires confirmation. At present it does not appear possible to make this prediction simply and reliably in individual patients.

It is clearly very important that the appraisal of a patient's need and suitability for LTOT should be made when the clinical condition is stable, as recommended in the DHSS draft guidelines, and not during or shortly after an exacerbation of the disease. Patients are often first referred to hospital in the course of such an exacerbation, when typically they are oedematous and their blood gas tensions are worse than usual. Throughout the period of assessment the patients should continue to receive whatever medication is appropriate and at the optimum dosage to minimise their airflow obstruction and any fluid retention. During this time arterial hypoxaemia would be confirmed and then shown to be persistent after a suitable interval; three weeks (the recommended minimum) or a month is convenient. The arterial carbon dioxide tension and $\mathrm{pH}$ are also recorded as well as the results of pulmonary function tests. A chest radiograph, an electrocardiogram and measurement of the haematocrit would normally be required for a full assessment. Few would disagree with Petty that measurement of the pulmonary arterial pressure is not required in deciding whether to treat or not. ${ }^{19}$ Neither are studies during sleep necessary, although it has been suggested that hypoxaemia at this time may be of particular relevance to the development of pulmonary hypertension. ${ }^{2021}$ What is essential is to confirm, firstly, that oxygen therapy raises the patient's arterial oxygen tension above $60 \mathrm{~mm} \mathrm{Hg}(8 \mathrm{kPa})$ and, secondly, that it does not aggravate carbon dioxide retention.

Adequate oxygenation is achieved in the majority of subjects with a flow of 1-3 litres of oxygen per minute, ${ }^{17}$ delivered by nasal cannulae. Several different designs of lightweight cannulae are now available which are well tolerated when worn for many hours at a time. Occasionally a patient finds the 
prongs of the cannulae too uncomfortable in the anterior nares and a controlled oxygen face mask must be worn instead.

It is well known that during exacerbations of respiratory failure some patients are intolerant of even modest increments in inspired oxýgen concentration and those with chronic stable hypercapnia may have a significant rise in arterial carbon dioxide tension within the hour when they are breathing high concentrations of oxygen. ${ }^{22}$ In patients selected for LTOT it must be shown that this does not occur overnight with the prescribed low flow supplement of oxygen and this needs to be done under supervision in hospital.

\section{Education of the patient}

The education of those selected for LTOT, and of a relative if necessary, requires particular attention. In addition to training the patient to use a concentrator of the same type that is to be installed at home, it is important to ensure that the objectives of treatment and the need for compliance are understood. Other aspects of patient education deserving attention might include the correct use of bronchodilators and the problem of smoking. Every effort should be made to persuade smokers to stop this habit before instituting LTOT; indeed, should they not be obliged to do so?

The siting of the concentrator in the patient's home should be discussed as well as the placement of oxygen outlets in the bedroom and living room. The most suitable location for the concentrator will be finalised by agreement between the installer and the patient; it should be a well ventilated, clean and dry location away from open fires or paraffin or gas heaters. The oxygen pipeline from the concentrator, for which heavy walled PVC tubing is quite satisfactory, can be routed relatively inconspicuously to the chosen outlet points; again the installer will attend to the final details of this. Arrangements for trouble shooting in the event of a fault developing with the concentrator also require explanation. Some patients who are totally or heavily dependent on their oxygen supply need a standby oxygen cylinder with attachments at home for emergency use in the event of a power failure or a concentrator fault which cannot be rectified promptly. Because of the pressure on hospital inpatient time and on busy clinics, educating patients is often an insufficiently fulfilled aspect of patient care. Who should be responsible here? While I do not wish to diminish the educational role of the physician, a good case can be made for an experienced and suitably trained nurse, or perhaps a physiotherapist or physiological measurement technician, to be dedicated to this important enterprise. The time which would be required for this task might not be long at first but it would surely increase as the number of concentrators in use grows. A service of this kind would be of more general value in the chest clinic and its provision could be linked with other duties. A suitably qualified "educator" could also play an active part in assessing patients who have been identified as candidates for LTOT, in determining the oxygen flow rate suitable for those selected for treatment, and in their future follow up, preferably at home as well as in the outpatient clinic.

\section{Compliance}

A patient's compliance with the advice and treatment recommendations given in hospital may well be enhanced by domiciliary follow up visits. In the NOTT trial ${ }^{5}$ very good. compliance was recorded with respect to the number of hours the patients used their oxygen supply, and a feature of that study was the frequency with which patients were visited at home by a nurse practitioner. It is likely that the NWRHA introductory trial of concentrators ${ }^{10}$ reflects more realistically what will happen with LTOT in routine practice in Britain. In that study 76 patients used their machines on average 14.3 hours daily, almost all having been instructed to do so for 15 hours or more. A similar observation was made by Evans, Waterhouse and Howard in a group of 14 patients. ${ }^{23}$ Moreover, in the NWRHA trial the frequency distribution of the number of hours per day that patients used their concentrators was negatively skewed; indeed six of the 76 patients were using their machines for less than six hours a day. Thus it appears that, if the necessary minimum daily duration of oxygen therapy for improved survival to accrue is in fact 15 hours, ${ }^{6}$ and the findings of the NWRHA study are representative of patient compliance in this country, then over half of the recipients will be having insufficient treatment. This is not to say that patients receiving oxygen for less than 15 hours a day derive no benefit from the treatment: their gain may be in the short term and symptomatic rather than in life expectancy. What is to be done when compliance is grossly inadequate? Should the patients constantly be exhorted "to do better'? Should treatment be abandoned, and would this be ethically sound? If the prospect of a failure in compliance could be predicted there would be no financial justification for installing a concentrator in the patient's home; a cylinder supply would be appropriate for the occasional user consuming oxygen at a rate of two litres per minute for no more than three or four hours daily. If poor compliance is anticipated it would seem reasonable to start treat- 
ment using cylinders and to switch to a concentrator later only if this is justified.

The availability of oxygen concentrators will permit those who retain lingering doubts about the benefits of LTOT to observe the treatment in practice. A combination of careful and sensitive assessment on the one hand and well informed and repeated guidance on the other could ensure that the machines are deployed and used appropriately throughout the country so that abuse of long term oxygen therapy, which is currently causing concern in the United States, ${ }^{24}$ can be avoided.

T B STRETTON

Manchester Royal Infurmary Manchester M13 9WL

\section{References}

1 Williams BT. Geographical variations in the supply of domiciliary oxygen. Br Med J 1981;282:1941-3.

2 Jones MM, Harvey JE, Tattersfield AE. How patients use domiciliary oxygen. $\mathrm{Br}$ Med J 1978;1:1397-1400.

3 Stark RD, Finnegan P, Bishop JM. Daily requirement of oxygen to reverse pulmonary hypertension in patients with chronic bronchitis. $\mathrm{Br} M e d \mathrm{~J}$ 1972;3:724-8.

4 Anderson PB, Cayton RM, Holt PS, Howard P. Longterm oxygen therapy in cor pulmonale. Quart J Med N.S. 1973;42:563-73.

5 Nocturnal Oxygen Therapy Trial Group. Continuous or nocturnal oxygen therapy in hypoxemic chronic obstructive lung disease. Annals Intern Med 1980;93:391-8.

6 Report of the Medical Research Council Working Party. Long-term domiciliary oxygen therapy in chronic hypoxic cor pulmonale complicating chronic bronchitis and emphysema. Lancet 1981;1:681-5.

7 Heaton RK, Grant I, McSweeney AJ, Adams KM, Petty TL. Psychological effects of continuous and nocturnal oxygen therapy in hypoxemic chronic obstructive pulmonary disease. Arch Intern Med 1983;143: 1941-7.

8 Timms RM, Khaja FU, Williams GE, and the Nocturnal Oxygen Therapy Trial Group. Hemodynamic response to oxygen therapy in chronic obstructive pul- monary disease. Annals Intern Med 1985;102:29-36.

9 Lowson KV, Drummond MF, Bishop JM. Costing new services: Long-term domiciliary oxygen therapy. Lancet $1981 ; 1: 1146-9$.

10 North Western Regional Health Authority Report No. 4/9/176. Pilot development of domiciliary oxygen concentrators. Manchester: NWRHA, December 1983.

11 Cooper AG. Oxygen production for medical use. Hospital Engineer 1968;12:85.

12 Cotes JE, Douglas-Jones AG, Saunders MJ. A 60\% oxygen supply for medical use. $\mathrm{Br}$ Med J 1969;4:1436.

13 Stark RD, Bishop JM. New method for oxygen therapy in the home using an oxygen concentrator. $\mathrm{Br} M e d \mathrm{~J}$ 1973;2:105-6.

14 Gould GA, Scott W, Hayhurst MD, Flenley DC. Technical and clinical assessment of oxygen concentrators. Thorax 1985;40:811-6.

15 Johns DP, Rochford PD, Streeton JA. Evaluation of six concentrators. Thorax 1985;40:000-000.

16 Williams BJ, Nicholl JP. Prevalence of hypoxaemic chronic obstructive lung disease with reference to long-term oxygen therapy. Lancet 1985;2:369-72.

17 Timms RM, Kvale PA, Anthonisen NR, Boylen CT, Cugell DW, Petty TL, Williams GW. Selection of patients with chronic obstructive pulmonary disease for long-term oxygen therapy. JAMA 1981;245:2514-5.

18 Ashutosh K, Mead G, Dunsky M. Early effects of oxygen administration and prognosis in chronic obstructive pulmonary disease and cor pulmonale. Am Rev Respir Dis 1983;127:399-404.

19 Petty TL. Who needs home oxygen? Am Rev Respir Dis 1985; 131:930-1.

20 Flenley DC. Clinical hypoxia: causes, consequences and correction. Lancet 1978;1:542-6.

21 Block AJ, Boysen PG, Wynne JW. The origins of cor pulmonale: a hypothesis. Chest 1979;75:109-10.

22 Rudolph M, Turner JA, McM, Harrison BDW, Riordan JF, Saunders KB. Changes in arterial blood gases during and after a period of oxygen breathing in patients with chronic hypercapnic respiratory failure and in patients with asthma. Clinical Science 1979;57:389-96.

23 Evans TW, Waterhouse J, Howard P. Clinical experience with the oxygen concentrator. $\mathrm{Br} \mathrm{Med} J$ 1983;287:459-61.

24 Greentree LB. Home oxygen therapy: a proposal. Am Rev Respir Dis 1985;131:932-3. 